\title{
Psychological treatments course for senior registrars in child psychiatry
}

\author{
Alicia Etchegoyen and Diana Cassell
}

\begin{abstract}
We have witten this paper from the perspecttve of a trainer and a trainee who have each participated in the poychological treatments course run for senior registrars training in child poychiatry on the North West Thames Rotation. The course aims to provide senior registrars with an integrated training in the three major poychological theraples: systemic family therapy, cognifivebehavioural and poychodynamic therapy. It was hoped that, by the end of the course, trainees would hove a good theoretical and clinical grounding in each model at a medium lovel of expertise.
\end{abstract}

\section{The course}

The course is divided into modules of one year for each treatment modality. Each module is organised by a consultant child psychiatrist with considerable clinical experience and advanced training in that particular modality. One of the trainers also acts as the co-ordinator for the whole course. The three course leaders meet once a term to review progress and to integrate ideas.

The course is open to child and adolescent psychiatry senior registrars in the region (the rotation grew during this period from six to ten trainees) and to other professionals with a similar level of training. Initially a whole morning was allocated to it, but from the second year this has been reduced to two and a half hours because of pressure on the senior registrars' time.

The course starts with the systemic family therapy module run by a consultant child psychiatrist (Dr Peter Reder) and a principal clinical child psychologist (Ms Sylvia Duncan). The first term includes learning about systemic ideas, such as the family life cycle and use of the genogram, and basic techniques such as circular questioning and co-therapy; the second term in applying these to clinical situations and therapy sessions; the third term allows for more clinical practice as well as consideration of consultations to other professionals and to multi-agency networks.
The cognitive-behavioural module follows. It is run by a consultant child psychiatrist (Dr Veira Balley) with invited speakers throughout the year. The first term is for learning behavioural therapy and introducing the cognitive model. The second term is spent in developing techniques (such as the use of thought diaries and schema elucidation) and their clinical applications. The third term covers research into efficacy and the use of cognitive therapy in particular situations such as following traumatic events.

The third year is for psychodynamic therapy. and is run by one of the authors (AE). The first term is spent on observational studies of a baby or young child, (McFadyen, 1991). The second term focuses on communication with children and the third on assessment, with outside speakers. The third term finishes with an integration of the three therapy models, organised as a series of dialogues.

\section{A trainee's experiences}

Prior to the course I (DC) had worked in settings which offered 'hands-on' training in behaviour modification and family therapy, and I had seen patients for supervised dynamic psychotherapy. I welcomed the opportunities to train in cognitive therapy and to do supervised infant observations which are not generally available during senior registrar training. It was sometimes hard making time for the extra reading but worth the effort.

In comparison with trainees from other regions, I am aware that this was an unusual and valuable course which extended my skill development beyond a level normally offered by case supervision and academic teaching, because the discussions were of high quality, more reflective and included more clinically-focused theoretical input. I feel I have gained a good level of experience in all three modalities and can integrate models in order to assess, select and connect different components of therapy. I valued the leaders' model of openness to other approaches combined with rigour in applying their own particular approach. 


\section{Evaluation of the course}

We sent a questionnaire to the senior registrar trainees asking about their experience of the course. Thirteen had attended part or all of the course, 11 replies. Ten would recommend such a course to a newly-appointed senior registrar (because they valued the combination of theory and clinical learning).

Trainees believed they had benefited as follows:

nine, consolidated skills

eight, learnt new skills

eight, professional growth/development

seven, future job prospects/CV improved

five, improved communication within multidisciplinary teams.

Additional comments focused on anxieties about pursuing further training in other centres; five reported encountering difficulties ensuring adequate clinical experience concurrent with course modules, due to the specialist setting of their posts. Constructive criticisms included having a more experiential format, live case work, considering a consultation using each modality, and a comment that it was unhelpful when other colleagues had joined the course but did not attend regularly. There was a suggestion to link it to a higher qualification such as an MSc.

\section{A trainer's experiences}

I found participation in the overall planning and teaching of the course exciting and demanding. Running the course took more time and effort than anticipated, especially the planning meetings and organising the infant observations. Much expertise and flexibility were required to teach appropriately to a group with varied levels of psychodynamic knowledge.

It was, for me, a valuable experience to work closely with colleagues who are expert using other treatment modalities. The dialogues with pairs of the course leaders were particularly instructive. The integration module started when an experiential exercise, which provided questions for discussion:

How do fundamental concepts in each model overlap or differ (e.g. transference, systems and cognitions)? Are they 'translatable"?

How to formulate a clinical situation from psychodynamic, systemic and cognitivebehavioural perspectives?

Can different techniques be used together? How and when to decide on which approach?

More abstract questions evolved, such as what constitutes psychological change, the nature and validity of psychological knowledge and the role of emotions in human behaviour.

\section{Conclusions}

We believe that this course is a valuable addition to a higher psychiatric training scheme because it guarantees a comprehensive and balanced training at an above average level of expertise.

The Royal College of Psychiatrists recommends that psychiatrists should be exposed to a range of clinical experiences (Joint Committee on Higher Psychiatric Training, 1987) during their training which occurs in a series of short placements, not usually planned around therapeutic models. The development of diagnostic and research skills may become prioritised. We have also found that the treatment models used, and the level of expertise in teaching them, varies between child psychiatry departments. As a result we believe that comprehensive training in therapeutic skills is unlikely to be available to the majority of trainees without a specific course.

There have been concerns about the future viability of such a course, given the trainers' need to account for time spent, leading to the possible introduction of a fee. Trainees worry that this may preclude study leave payments to attend other conferences.

We think that this type of course has great training value for trainees in adult psychiatry (and other professionals) too. The College's recent guidelines for training in general psychiatry (1993) recommend that psychotherapy training should be mandatory and that trainees should have supervised experience in the three treatment modalities discussed here. Our course would make a positive contribution towards fulfilling these training requirements and so the time involved would be justified.

\section{References}

MCFADYEN, A. (1991) Some thoughts of infant observation and its possible role in child psychiatry training. Newsletter of the Association for Child Psychology and Psychlatry. 13, 10-13.

Royal College OF PSYCHIATISTS (1987) Jotnt Committee on Higher Psychiatric Training Handbook: Section 2 Training Requirements.

- (1993) Guldelines for psychotherapy training as part of general professional psychiatric training. Psychiatric Bulletin, 17, 695-698.

Alicia Etchegoyen, Consultant Child Psychiatrist, Chelsea and Westminster Hospital, Fulham Road, London SW10 9TH; and Diana Cassell, Consultant Child Psychiatrist, Child, Adolescent and Family Centre, 84 Ewell Road, Surbiton, Surrey KT6 6EX 